\title{
Cardiopatía y embarazo
}

Clara Isabel González Moncada

Gerardo Blass Alfaro

Unidad de ARO, Hospital Bertha Calderón Roque

g.blass@hotmail.com

Fecha de recibido: 6 de agosto de 2015

\section{RESUMEN}

Paciente de 23 años de edad, primigesta, con 32 semanas de gestación, portadora de cardiopatía congénita (conducto arterioso persistente de $0.7 \mathrm{~mm}$ de diámetro) diagnosticada a los 10 años de vida, con cortocircuito de izquierda a derecha quien además presenta dilatación de cavidades izquierdas y datos de Hipertensión pulmonar leve. Desde el inicio del embarazo ha cursado asintomática y compensada. Al examen físico se encontró soplo IV/VI en todo el borde esternal izquierdo irradiado a foco aórtico y pulmonar, con ausencia de plétora yugular y de cianosis peribucal. Se le practicó cesárea y esterilización quirúrgica, previo consentimiento informado, a las 38 semanas de gestación bajo anestesia regional, obteniéndose un feto vivo, apgar $8 / 9$, sin complicaciones asociadas y con evolución satisfactoria de su puerperio

Palabras clave: Conducto arterioso persistente, Hipertensión pulmonar.
Fecha de aprobado: 12 de septiembre de 2015

\section{ABSTRACT}

Patient 23 years old, first pregnancy, with 32 weeks of gestation, with congenital heart disease (patent 10 years old, wiht short left and right who also has dilated left chambers data and mild pulmonary hypertension. Since the beginning of pregnancy has completed asymptomatic compensated. Physical examination murmur IV/VI was found around the left sternal border radiating to the aortic and pulmonary focus with no jugular plethora and cianosis. He underwent cesarean section and surgical sterilization prior informed consent, at 38 weeks of gestation under regional anesthesia, obtaining a live fetus, Apgar 8/9, without associated complications and with satisfactory evolution of their postpartum.

Keywords: patent ductus arteriosus, pulmonary hypertension

\section{INTRODUCCIÓN}

El ductus arterioso (DA) es una estructura vascular que conecta la aorta descendente proximal con la arteria pulmonar principal cerca del origen de la rama pulmonar izquierda. Es esencial para la vida fetal, se cierra espontáneamente después del nacimiento en la mayoría de los recién nacidos a término (RNT). Sin embargo, en los prematuros el cierre del ductus se produce con frecuencia más allá de la primera semana de vida, especialmente en aquellos que precisan ventilación mecánica. 1

En el RNT el hallazgo de ductus arterioso permeable (PDA) generalmente está relacionado con un defecto anatómico del ductus o de otras partes del corazón. Aunque la mayoría de los casos ocurren de forma esporádica es importante resaltar el papel, cada vez más relevante, de los factores 
genéticos y de las infecciones prenatales, como la rubeola cuando se padece en las primeras cuatro semanas del embarazo. También hay una mayor incidencia asociada a teratógenos (alcohol, anfetaminas, anticonvulsivan-tes, fenitoína). Existen casos asociados a cromosopatías (+14q, $\mathrm{XXY)}$ y se piensa que existe una base hereditaria multifactorial. 1, 2

\section{REPORTE O DESCRIPCIÓN DEL CASO}

Se trata de Paciente de 23 años de edad, femenina, mestiza, ama de casa, procedente de Bluefields. En relación a sus factores de riesgo durante el embarazo se identifica persistencia del conducto arterioso grande diagnosticada por ECO-BD el 13/04/2000 (ver imágenes de ECO de controles figura 1 y figura 2) y P/E como APF. Paciente quien ha cursado asintomática desde el momento de su diagnóstico, con buen estado general, con exámenes de laboratorio dentro de parámetros normales durante todos sus controles prenatales, sin comorbilidades y con evolución clínica silente. Paciente con Diagnostico ya conocido, fue trasladada el día17/07/13 del Hospital de Bluefields al Hospital Bertha Calderón Roque con 32 2/7 SG por US para su manejo y tratamiento.

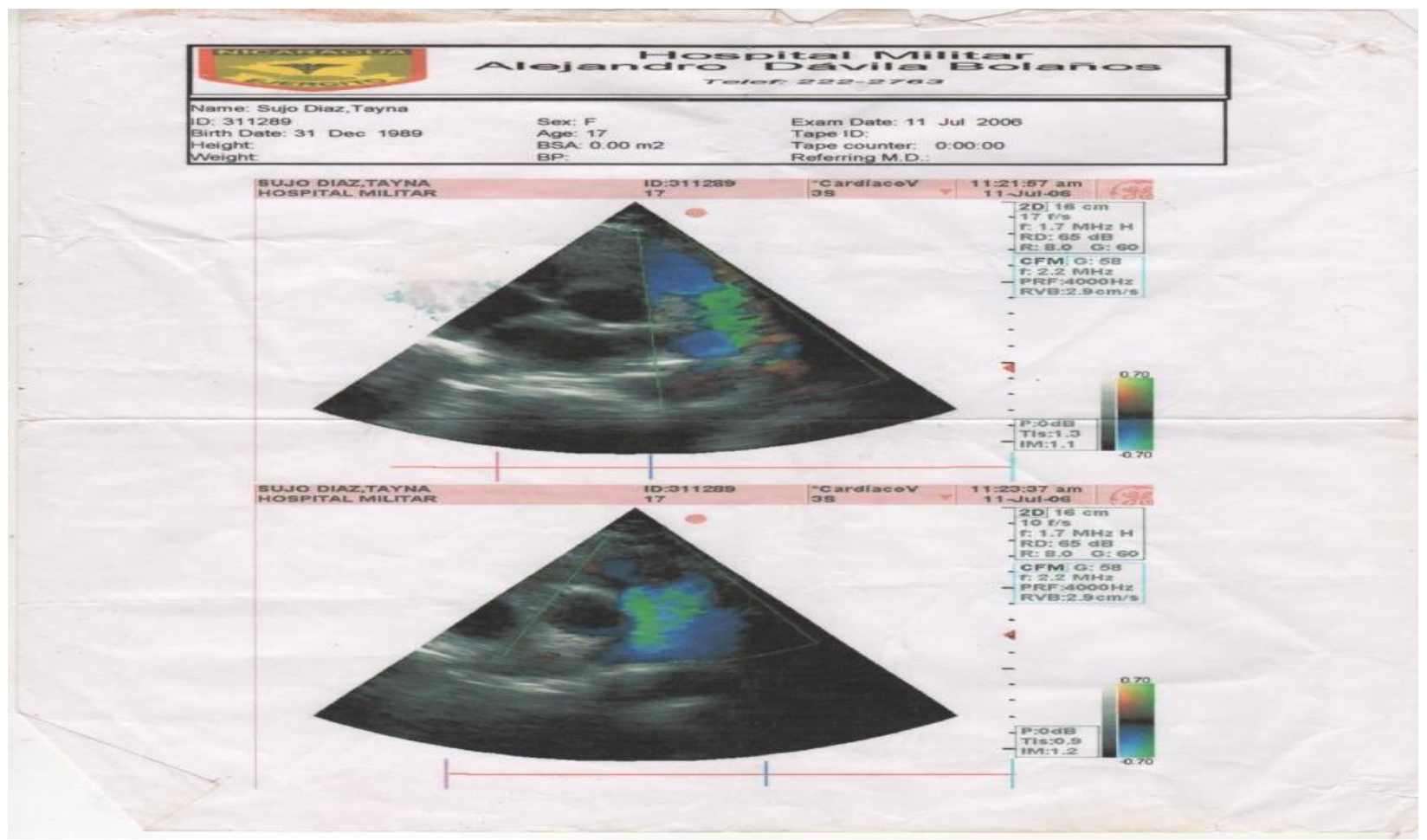

Fig 1. Ecocardograma en el que se evidencia: 1- Ligera dilatación de cavidades izquierdas, el patrón de contractilidad de ventrículo izquierdo es normal en reposo, la fracción de eyeccion es normal. 2- Cavidades dereche de tamaño normal, no se observa derrame pericárdico. 3- En la vista eje corto transversal para grandes vasos con doppler a color se visualiza flujo que pasa de la Aorta a la arteria pulmonar derecha a traves de la persistencia del ductus arterioso. Leve incremento de las cifras de presión sistólica de la arteria pulmonar. Diagnosis: DUCTUS ARTERIOS PERSISTENTE 
Al examen físico a su ingreso se encuentra:

- Cabeza y cuello: no plétora yugular, no cianosis peribucal

- Tórax: simétrico, expansible

- Campos Pulmonares: M/V audible, no se auscultan ruidos patológicos agregados

- Cardíaco: ritmo sinusal, de buen tono, se ausculta soplo IV/VI en todo el borde esternal izquierdo que se irradia a focos aórtico y pulmonar.

- Abdomen: globoso, peristalsis audible, con útero ocupado por feto único, vivo, eutócico, longitudinal, dorso izquierdo, con FCF de 140-148, AFU: $30 \mathrm{cms}$

- Neurológico: conservado

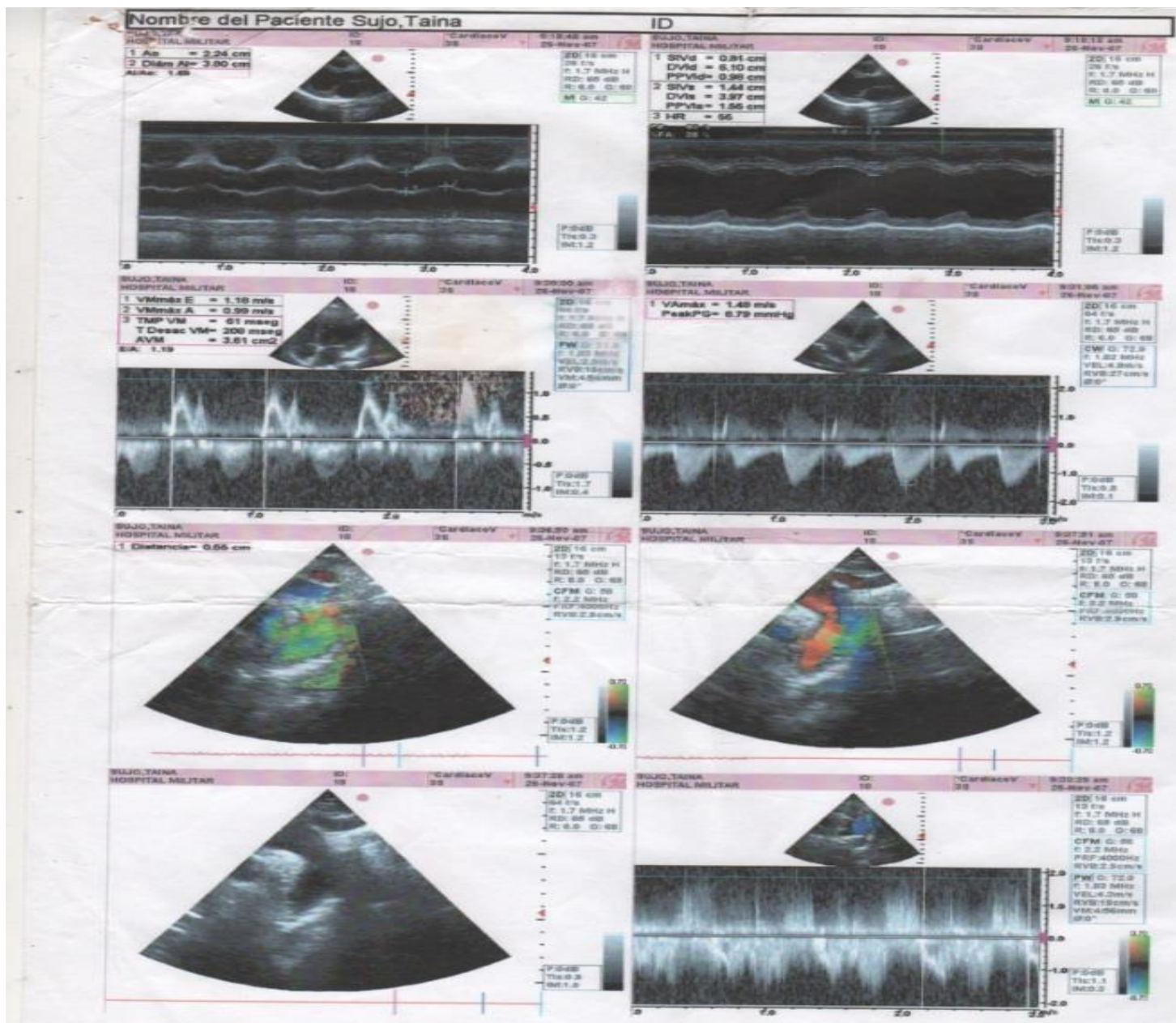

Fig. 2. Ecocardiograma que reporta: Cavidades izquierdas dilatadas, grosor normal, sin alteraciones de la movilidad global y segmentaria, sin alteraciones de la relajación isovolumetrica, patrón de llenado tipo normal. Funion sistólica normal. Valvulas mitral, 
aortica y tricúspide normales. Presencia de conducto arterioso permeable de $0.7 \mathrm{~mm}$ de diámetro. Diagnosis: PERSISTENCIA DEL CONDUCTO ARTERIOSO.

A su ingreso comienza a aquejar disnea de medianos esfuerzos. En dicha unidad se le inicia tratamiento con carvedilol a dosis de $3.125 \mathrm{mg}$ P.O c/ $12 \mathrm{hrs}$, profilaxis antitrombotica con Heparina a dosis de 5,000 uds S.C c/ 12 hrs, además de sus fármacos, cuidados y medidas generales como son: su esquema de maduración pulmonar, Sulfato ferroso + ácido fólico, líquidos P.O restringidos a $800 \mathrm{cc}$ por ASC (3600 cc P.O c/ 8 hrs) y O2 por razones necesarias. Paciente cursando 11 días de estancia intrahospitalaria, quien ha evolucionado satisfactoriamente, sin repercusiones hemodinámicas, sin presentar reacciones adversas medicamentosas, cuya vía de finalización del embarazo será cesárea a las 38 SG previendo las repercusiones hemodinámicas y las complicaciones debidas al sobreesfuerzo del parto por vía vaginal que podría presentar la paciente en el periparto y puerperio inmediato.

\section{DISCUSIÓN}

Esta anomalía es rara en adultos por su corrección quirúrgica precoz. Su persistencia ocasiona cortocircuito de izquierda a derecha de magnitud variable y en raros casos hipertensión pulmonar e inversión del flujo con Sindrome de Eisenmenger. Durante el embarazo, todas estas alteraciones que acompañan al PDA suponen un riesgo mayor para descompensación hemodinamica. 3

De acuerdo a todos los cambios fisiológicos que se producen durante el embarazo, los mayores peligros para una embarazada cardiópata se presentan:

- entre las 20 y 24 semanas de embarazo

- durante el trabajo de parto y parto y

- $\quad$ en el post-parto inmediato. 4

En general, los cambios cardiovasculares asociados con la gravidez retornan a la normalidad dentro de los 6 meses del post-parto, aunque la mayoría de estas regresiones ocurren dentro de las 2 semanas. 4

La gestación afecta a la cardiopatía, sobrecargando la reserva funcional del corazón, de modo que se considera que empeora un grado de la clasificación funcional de la New York Heart Association (NYHA) durante la gestación (Tabla 1). Esta clasificación funcional se asocia con la 
morbimortalidad materna, las pacientes en grado III-IV tienen peor pronóstico. El tipo de cardiopatía es fundamental para individualizar el riesgo que conlleva la gestación. En la Tabla 2 se muestra una clasificación de las cardiopatías en función de la mortalidad materna asociada. Algunas situaciones son indicación de interrupción de gestación por alto riesgo materno (Tabla 3).

Tabla 1. Clasificación funcional de la gestante cardiópata según NYHA.5

Limitación $\quad$ Síntomas (fatiga, palpitaciones,
Actividad

\begin{tabular}{|r|l|l|l|}
\hline \multicolumn{1}{r}{ Clase I } & No & Con ejercicio intenso & \\
\cline { 2 - 4 } Clase III & Moderada & Con ejercicio habitual & $0.1 \%$ \\
\cline { 2 - 4 } Clase IV & Important e & Con ejercicio leve & $0.3 \%$ \\
\cline { 2 - 4 } & & & $5.5 \%$ \\
\cline { 2 - 4 } & & En reposo & $6 \%$ \\
\hline
\end{tabular}

Tabla 2. Grupos de riesgo de mortalidad materna.5

\begin{tabular}{|c|c|c|}
\hline Grupo & Patologia & Mortalidad \\
\hline$I$ & $\begin{array}{l}\text { Comunicación interauricular Comunicación } \\
\text { interventricular no complicada PDA no } \\
\text { complicada Enfermedad } \\
\text { pulmonar/Tricuspidea } \\
\text { Fallot corregido Valvula Biologica }\end{array}$ & Menor 1\% \\
\hline II & $\begin{array}{l}\text { Estenosis mitral } \\
\text { Estenosis aortica } \\
\text { Coartacion de aorta no complicada } \\
\text { Fallot no corregido } \\
\text { IAM previo } \\
\text { Válvula mecánica }\end{array}$ & $5-15 \%$ \\
\hline III & $\begin{array}{l}\text { Sindrome de Marfan } \\
\text { Hipertension pulmonar } \\
\text { Coartacion aorta complicada }\end{array}$ & $25-50 \%$ \\
\hline
\end{tabular}


Tabla 3. Indicaciones de interrupción de embarazo.5

Hipertensión Pulmonar

Cardiomiopatía dilatada

Síndrome de Marfan con afectación cardiovascular

Fistula auriculoventricular pulmonar

Cualquier lesión no corregible en fases III-IV

El signo clínico más frecuente es un soplo sistólico de eyección, con menos frecuencia continuo, que se ausculta mejor en región infraclavicular izquierda y borde paraesternal superior izquierdo que con frecuencia se irradia al dorso. La aparición del ductus se puede acompañar de: Precordio hiperactivo, taquicardia, pulsos saltones en región postductal, polipnea, apnea, hepatomegalia.

\section{Control durante la gestación:}

- Control multidisciplinario: cardiólogo y obstetra. Las visitas deben ser cada 4 semanas hasta la 23, cada 2 semanas hasta la 33, y cada semana hasta el ingreso.

- Debe realizarse una valoración del estado funcional de la paciente (NYHA), en cada visita de la gestación.

- Restringir:

- actividad física, reposo en cama al menos 12 horas diarias

. humedad, calor, ansiedad, alcohol, tabaco

- Suplementos: hierro y ácido fólico (anemias) 
- Dieta hiposódica. No más 7-9 kg de peso ganancia final

- Prevenir éstasis venoso (medidas posturales, medias compresión)

- Vigilar infecciones intercurrentes

- Controlar potasio, sodio y hematocrito

- Tratamiento cardiopatía.5

\section{Control durante el parto}

En cuanto a la finalización de la gestación, la decisión de cuándo y cómo concluirla depende del equilibrio de los trastornos maternos y fetales. Hay que considerar la evolución del estado funcional con respecto al tiempo.

- Parto espontáneo a término: clases funcionales I y II sin indicaciones fetales de terminación.

- Parto inducido semanas 38-39: clases funcionales

\section{III-V. 5}

\section{Vía del parto}

El parto vaginal no complicado es mejor tolerado a nivel hemodinámico que la terminación por cesárea, por lo que es la vía de elección en la mayoría de estas pacientes, excepto:

- Estenosis aórtica severa

- Dilatación aórtica > 45 mm o disección aórtica

- IAM reciente

- Reacción de Eisenmenger

- Endocarditis aguda que requiere cambio valvular

- Descompensación aguda grave. 5

\section{Control durante postparto}

Tras el parto, la situación hemodinámica continúa siendo de riesgo para la paciente, precisando en los casos de alto riesgo seguimiento en unidades de cuidados intensivos, al menos las primeras 48 horas. 
- Primeras horas

- $\quad$ Oxitocina IV no muy rápida pues produce hipotensión

- $\quad$ No usar ergotínicos por sus repercusiones hemodinámicas

- $\quad$ Mayor incidencia de hemorragia en pacientes de alto riesgo (Marfan, clases funcionales IIIIV, cianógenas), preparar posible transfusión - Iniciar heparina en casos seleccionados en 4-6 horas, a la vez que dicumarínicos

- Primeros días

- Movilización precoz

- Medias elásticas

- No hay contraindicación específica para la lactancia, la mayoría de medicamentos pueden utilizarse concomitantemente. 5

\section{CONCLUSIONES Y RECOMENDACIONES}

Las pacientes con cardiopatía conocida deben ser informadas sobre las complicaciones de la gestación desde el comienzo de su etapa fértil.

Sobre algunas patologías, especialmente cardiopatías congénitas complejas con reparación quirúrgica, la experiencia obstétrica es anecdótica.

La cardiopatía representa un riesgo potencial tanto para la madre como para el feto, a pesar de ello, no todas las pacientes embarazadas cardiópatas tendrán complicaciones durante su gestación.

Un manejo oportuno y adecuado por parte del personal médico es determinante para garantizar el bienestar materno-fetal.

\section{REFERENCIAS}

Ruiz González, Ma, Guzmán, Gómez, P.M, Tejero, M., Guzmán, J. Ductus arterioso persistente. Unidad de Neonatología, Unidad de Cardiología Pediátrica .H.U. Reina Sofía Córdoba. Cap. 36. 
Medrano, C y Zavanella, C. Ductus arterioso persistente y ventana aorto pulmonar. S. de Cardiología Pediátrica y Cirugía Cardíaca Hospital Juan Canalejo. La Coruña.

Shwarcz, R.; Fescina, R.; Duverges, C. (2011). Obstetricia. Buenos Aires. Sexta edición.

Cattaneo, A. Manejo Anestésico de la Embarazada Cardiópata. Argentina. Volumen 16 Suplemento 12004 Sego. Fundamentos de Obstetricia. 